\title{
PERCEPCIÓN DEL CLIMA ORGANIZACIONAL EN ATENCIÓN PRIMARIA DE SALUD EN LA REgIÓN DEL MAULE, CHILE
}

PERCEPTION OF THE ORGANIZATIONAL CLIMATE IN PRIMARY ATTENTION OF HEALTH IN THE REGION OF THE MAULE, CHILE

\author{
Francisca Pilar Adriazola Díaz, ${ }^{1}$ María Josefa López Hermosilla, ${ }^{1}$ Rodrigo Andrés Rojas Pérez, ${ }^{1}$ \\ Miguel A. Bustamante U. *2
}

1 Magíster en Gestión de Sistemas de Salud, Universidad de Talca, Chile, Dos Norte 685, 3465548 Talca, Chile.

2 Facultad de Economía y Negocios, Universidad de Talca, Avenida Lircay s/n, Talca, Región del Maule, Chile.

E-mail: fadriazola@utalca.cl ; josefa.I.h@gmail.com ; rrojas@csmaule.com; mabu@utalca.cl

RESUMEN

ABSTRACT

Este trabajo mide el clima organizacional de cuatro centros de Atención Primaria de Salud de cuatro comunas de la Región del Maule, Chile. Se aplicó el Cuestionario de Clima Organizacional Validado (CCOV) de manera censal a funcionarios de seis centros asistenciales APS totalizando 389 casos. La confiabilidad del instrumento fue alta y se realizaron análisis demográficos, de factores exploratorios AFE y análisis inferencial. La dimensión que más afecta el clima organizacional es Equipos y distribución de personas y material; en tanto que la dimensión que contribuye positivamente con el clima es Autonomía y Profesionalismo. El Cuestionario se concentró en siete factores y 30 reactivos y permite la elaboración de sugerencias de mejora del clima organizacional en APS.

PALABRAS CLAVE: Clima organizacional; Institucio-

This work measures the organizational climate of four centers of Primary Attention of Health of four communes of the Region of the Maule, Chile. The Questionnaire of Validated Organizational Climate was applied (CCOV) of way censal to officials of six assistance centers APS totalling 389 cases. The dependability of the instrument was high and they were carried out demographic analysis, of exploratory factors AFE and analysis inferencial. The dimension that more it affects the organizational climate it is Teams and people's distribution and material; as long as the dimension that contributes positively with the climate is Autonomy and Professionalism. The Questionnaire concentrated on seven factors and 30 reagents and it allows the elaboration of suggestions of improvement of the organizational climate in APS.

KEYWORDS: Organizational climate; Institutions of health; Dimensione of the organizational climate. nes de salud; Dimensione del clima organizacional 


\section{Introducción}

El Sector Salud es el proveedor básico de esta necesidad social por parte del estado (OMS, 2006; 2008) y dado que ha sido uno de los más requeridos, hace de él un espacio de alta complejidad (Bustamante y Opazo, 2004) que requiere gestionar el clima de sus organizaciones, por una parte, para mejorar la satisfacción de los usuarios (Pelaes, 2010) y por otra, por ser especialmente relevante cuando chile enfrenta un proceso de envejecimiento acelerado (CEPAL. 2012; INE. 2014; SENAMA. 2013).

A nivel nacional e internacional se han realizado múltiples estudios de clima organizacional, algunos diagnosticando sus impactos sobre la calidad de servicios (Bernal, Pedraza \& Sánchez, 2015; Bustamante, Grandón \& Lapo, 2015; Bustamante, Lapo \& Grandón, 2016) y otros, analizando efectos sobre el entorno laboral y satisfacción de los prestadores (Greenslade \& Jummieson, 2011; Puch, Chávez \& Almeida, 2012; Segura, 2012) ratificando el hecho de que los ambientes laborales son vitales en el desarrollo de organizaciones complejas (Acevedo, Farías, Sánchez, Astegiano \& Fernández, 2012; Bustamante et al., 2004; Segredo, 2015, 2013; Urquiza, 2012), en tanto se busca mejorar los indicadores de calidad de servicio y eficiencia de los prestadores sanitarios (Jiménez, 2004).

El clima organizacional describe la atmósfera social de las organizaciones (Cardona \& Zambrano, 2014) e identifica a los directivos como responsables de crear un clima interno que influya de manera positiva en las conductas y cultura organizacional (Pettigrew, 1979) generando un sistema de significados colectivamente aceptados (Cújar, Ramos, Hernández \& López, 2014).

El clima interno se construye a partir de percepciones que no necesariamente reflejan la realidad tal cual es (Bustamante, et al., 2015; Bustamante, et al., 2016) sin embargo ayudan a distinguir a una institución de otra e incluso a determinar microclimas a su interior (Noboa, 2007).

En Suecia, se concluyó que puntuaciones altas del cuestionario de clima, en combinación con una estrategia de implementación explícita, predicen resultados positivos (Carlford, Andersson, Nilsen, Bendtsen \& Lindberg, 2010). Por su parte en Estados Unidos, el instrumento diseñado para medir el clima organizacional entre practicantes de enfermería en APS, obtuvo alta fiabilidad y consistencia (Poghosyan, Nannini, Finkelstein, Mason \& Shaffer, 2013). Finalmente en Chile, se elaboró un cuestionario con 14 dimensiones y 71 reactivos (Bustamante, et al., 2015) el cual fue posteriormente validado en dos centros de alta complejidad de la Séptima Región del Maule, dando origen al Cuestionario de Clima organizacional Validado (CCOV) que concentra 44 reactivos en 10 dimensiones (Bustamante, et al., 2016).

Se ratifica en consecuencia que medir el clima organizacional es una práctica que contribuye al mejoramiento continuo de las organizaciones (Bernal, et al., 2015), orienta la gestión directiva y mejora la toma de decisiones, trayendo consigo efectos notables en productividad, satisfacción personal, compromiso y calidad de servicio de los prestadores (Carmona \& Jaramillo, 2010; Neal, Griffin \& Hart, 200o; Pelaes, 2010; Vega, Arévalo, Sandoval, Aguilar \& Giraldo, 2006).

Complementariamente, se ha llegado a plantear que diagnosticar el clima organizacional contribuye a la excelencia, permite alcanzar ventajas competitivas y generar una cultura de servicio eficaz y eficiente para con la sociedad en general (Neal, et al., 2000) y que, en las instituciones de salud pública, permite orientar sus actuaciones hacia el cumplimiento de su misión (Patarroyo, 2012; Rodríguez, Álvarez, Sosa, De Vos, Gorbea \& Van der Stuyft, 2010).

Con todo, parece evidente que a nivel de APS, la asistencia sanitaria depende de la gestión de personas y de tecnologías cuyo eje central sea la salud (Vignolo, Vacarezza, Âlvarez \& Sosa, 2011), sin embargo, ante la existencia de déficit de profesionales (MINSAL, 2016; Téllez, 2016) es preciso fortalecer los sistemas sanitarios (Bass del Campo, 2012) mediante el diagnóstico del clima organizacional como una herramienta de primer orden en APS (Santa Eulalia et al., 2012) que sintetiza las percepciones de satisfacción (Pérez, Guillén-Crima, Brugos, Aguinaga \& Fernández-Martínez, 2012) y permite identificar cuáles factores influyen de manera fuerte o débil el clima interno y cómo estos contribuyen con eficacia a la misión (González-Burboa, Manríquez \& Venegas, 2014).

Sobre la base de lo señalado, el presente trabajo mide las percepciones del clima organizacional de los funcionarios de atención primaria de salud, APS para determinar cuáles dimensiones son las que más o menos inciden en sus conductas (Santa Eulalia y Sampedro, 2012; Senic \& Marinkovic, 2013) y busca responder a las preguntas de investigación: ¿Cuáles son las dimensiones que más inciden en el clima organizacional de los centros prestadores de salud a nivel de APS? y ¿Cómo se expresan y ponen de manifiesto, las variables que conforman el clima organizacional?

Es así como el estudio se estructura a partir de una síntesis conceptual seguida de la definición de objetivos y metodología pertinente. Se describe la validación de reactivos del cuestionario respecto de las dimensiones y reactivos definidos en el trabajo original (Bustamante, et al., 2016) y finalmente se detallan los resultados y presentan las conclusiones y hallazgos obtenidos en la investigación.

\section{Metodología}

A continuación se describe en forma resumida el contexto y la metodología de validación del instrumento y el trabajo de campo ejecutado para la medición del clima organizacional en APS. 


\section{Contexto APS}

Chile posee un sistema mixto de salud en el que se diferencian el sistema público y el privado, y de ambos el primero concentra cerca del $85 \%$ de la población (MINSAL, n.d.), con una rica historia en APS (Bass del Campo, 2012) dónde resalta una larga tradición de servicio y de transformación a partir del año 1997 que se expresó en un sistema descentralizado a nivel de las municipalidades y que ha permitido avanzar en cobertura universal y tener un sistema de salud con indicadores sanitarios al nivel de países desarrollados (Goic y Armas, 2003). Según la Ley 19.378 (1995) en su artículo 5, los funcionarios de Atención Primaria de Salud en Chile están clasificados en seis categorías (Ley 19.378, 1995) alcanzando una dotación en APS de 61.490 funcionarios (MINSAL, n.d.).

\section{Tipo de estudio}

Elpresenteestudiodefinidocomocuali-cuantitativo, observacional, transversal y censal, adoptó un carácter exploratorio para analizar el clima organizacional en centros de atención primaria de salud APS de la Séptima Región del Maule, Chile, mediante la descripción, análisis e interpretación de dimensiones (Eyssautier, 2006; Hernández, Fernández \& Baptista, 2006).

El Instrumento utilizado fue el Cuestionario de Clima Organizacional Validado CCOV (Bustamante, et al., 2016), que define 1o dimensiones con 44 ítems medidos mediante una escala Likert de 5 puntos; donde el valor 1 indica estar totalmente en desacuerdo con la afirmación y el valor 5 señala estar totalmente de acuerdo. La confiabilidad del instrumento se determinó por el Alfa de Cronbach, asumiendo un valor mínimo de o,8 para el análisis (Meliá, Pradilla, Martí, Sancerni, Oliver \& Tomás, 1990; Oviedo \& Campo-Arias, 2005; Patterson et al. 2005).

\section{Descripción del instrumento original}

El cuestionario elaborado por Bustamante, et al. (2016) contiene 10 dimensiones de clima organizacional que a continuación se describen.

La dimensión Colaboración y Buen Trato, destaca la colaboración entre pares y la ayuda, apoyo y trato agradable que los altos mandos pueden generar en el clima organizacional. Motivación Laboral, se refiere al compromiso de los trabajadores para con sus respectivos trabajos. Iniciativa Participativa, releva la importancia de la inclusión de las personas en el trabajo cotidiano y destaca cómo las nuevas ideas contribuyen al buen desempeño en tanto encuentren el respaldo de los superiores.

Complementariamente, el factor Desarrollo profesional, muestra cómo los sistemas de ascensos y promociones otorgan oportunidades, dando espacios para la concreción de habilidades y talentos que se expresan en la asigna- ción de responsabilidades incrementales y de confianza que los funcionarios valoran. Apoyo al emprendimiento, promueve y estimula la generación de nuevas ideas como componente clave del desempeño que los funcionarios.

Equipos y distribución de personas y material, refiere a los componentes de la productividad de los factores productivos y que se manifiesta en coherencia, eficacia y eficiencia de las prestaciones de salud. En tanto que la dimensión Organización y desempeño, dice relación con la organización y planificación de las obligaciones, a la división de tareas, funciones y responsabilidades, respecto de un sistema de recompensas y estímulos adecuados. Estabilidad laboral, por su parte, representa la valoración que los funcionarios hacen de la seguridad del empleo y su pertenencia a una unidad de trabajo.

Comunicación y desarrollo, se ocupa de poner de manifiesto la importancia de los sistemas formales de comunicación entre personas, servicios y unidades para que trabajen de forma articulada y armónica. Finalmente, Autonomía y Profesionalismo, destaca el valor de la libertad, la valoración de la autonomía y la necesidad de los funcionarios de contar con espacio $\mathrm{y}$ atribuciones, por una parte, que les permitan ejercer con independencia sus talentos y, por otra, que se reconozca el valor de la experticia.

\section{Procedimientos de validación}

Se realizó el piloteo del instrumento (Hernández, et al., 2006) mediante la aplicación del cuestionario a 15 funcionarios no pertenecientes a la muestra del estudio, como si fueran respondientes normales representativos del contexto de atención primaria objeto de la investigación.

Se verificaron aspectos lingüísticos, comprensión, lenguaje, formato, instrucciones y escalas utilizadas. Luego el CCOV (Bustamante, et al., 2016) fue revisado por un grupo de expertos conformado por 8 directores comunales de APS, quienes no hicieron mayores alcances a la versión del cuestionario confirmándose unidireccionalidad de las proposiciones, validación del lenguaje utilizado en las afirmaciones y ratificación de la consistencia del constructo según los modelos teóricos que sostienen cada uno de los reactivos (Eyssautier, 2006; Hernández, et al., 2006; Patterson, et al., 2005).

\section{Determinación de la Muestra y confi- dencialidad}

El CCOV se aplicó de forma intensiva, a una muestra de 389 casos durante una semana de noviembre de 2016 de manera censal a funcionarios de APS de 4 comunas de la Séptima Región: Talca, Maule, Pencahue y San Javier, en sus propias oficinas o lugares de trabajo por los propios investigadores.

Con el objetivo de asegurar la confiden- 
cialidad de la información, el análisis de los datos se realizó resguardando la identidad, tratando de manera agrupada los casos recogidos y con absoluta reserva de la información de los respondientes. En consecuencia, se optó por aplicar el cuestionario en forma anónima bajo el principio de consentimiento informado, en el cual se comunicó a los funcionarios sobre la regulación establecida por el Estado de Chile en la aplicación de cuestionarios (Ley 17.374, 1970; Ley-19.628, 1999).

\section{Procesamiento de los datos}

El análisis y procesamiento de los datos se realizó mediante el software estadístico SPSS versión 24 e incluyó el análisis descriptivo, definición demográfica de la muestra (Eyssautier, 2006) y análisis de confiabilidad mediante el Alfa de Cronbach (Oviedo et al. 2005; Meliá, et al., 1990), además se aplicó el Programa Factor para los Análisis de Factores Exploratorios AFE y mediante la estandarización de las medias (Walpole, Myers \& Myers, 1999) se compararon dimensiones $(-3 \mathrm{a}+3)$ donde los valores estandarizados con signo negativo resultan cuando la media de la variable es menor a la media general y con signo positivo si la media de la variable está por encima de la media general (Eyssautier, 2006).

\section{Resultados}

Los resultados que a continuación se detallan, contienen los hallazgos obtenidos en las diversas fases de la investigación dando cuenta de la ejecución exhaustiva del método previamente descrito.

\section{Análisis descriptivo de la muestra}

Del total de funcionarios encuestados (389), el $71 \%$ corresponde a mujeres, el $45,8 \%$ tiene entre 26 y 35 años, el $47,6 \%$ tiene educación superior completa, el $31,9 \%$ tiene educación técnica completa.
Además, el 57,6\% es soltero, el $35 \%$ es casado. En términos socioeconómicos, el $32,6 \%$ pertenece a la categoría de ingresos medios C y el $32,1 \%$ pertenece a la categoría de ingresos bajos $\mathrm{B}$.

En términos laborales, el $49,1 \%$ tiene entre uno a cinco años de servicio, el $25,2 \%$ tiene de seis a once años de servicio. Complementariamente, el $55,5 \%$ tiene contrato indefinido y el $33,2 \%$ tiene contrato a plazo fijo.

\section{Confiabilidad del instrumento}

Como primer paso metodológico, se determinaron los coeficientes de confiabilidad para cada una de las 10 dimensiones (Tabla 1), alcanzando un Alfa de Cronbach general de 0.95 considerado muy bueno.

El segundo paso fue la obtención de los índices de confiabilidad de cada una de las dimensiones del instrumento. Las dimensiones que presentan una buena fiabilidad del instrumento fueron: Colaboración y Buen Trato, Motivación Laboral, Comunicación y Desarrollo, Iniciativa Participativa, Organización y Desempeño, Estabilidad Laboral, Equipos y Distribución de Personal y Material, todas ellas con un Alfa de Cronbach superior a o,8 (Oviedo et al., 2005).

Las dimensiones que presentan una moderada confiabilidad fueron: Autonomía y Profesionalismo y Apoyo al Emprendimiento, ambas con un Alfa de Cronbach superior a 0,5 e inferior a 0,8 .

Finalmente, la dimensión que presenta confiabilidad baja fue Desarrollo Profesional con un Alfa de Cronbach inferior a 0,5 .

La confiabilidad general del instrumento y la respectiva de cada una de las dimensiones permite confirmar (Tabla 1) que el cuestionario posee las características suficientes como para garantizar consistencia y validez de las mediciones de clima organizacional en APS en las cuatro comunas seleccionadas de la Región del Maule, Chile.

Tabla 1 Confiabilidad y promedio de las dimensiones

\begin{tabular}{|c|c|c|c|c|}
\hline \multirow[b]{2}{*}{$\mathrm{N}^{\circ}$} & \multirow[t]{2}{*}{ Confiabilidad } & \multirow[b]{2}{*}{ Alfa de Cronbach } & \multicolumn{2}{|c|}{ Promedios } \\
\hline & & & Promedio & Estandarización \\
\hline 1 & Colaboración y buen trato & 0,920 & 3,54 & 0,20 \\
\hline 2 & Motivación laboral & 0,920 & 3,57 & 0,23 \\
\hline 3 & Iniciativa participativa & 0,890 & 3,26 & $-0,02$ \\
\hline 4 & Desarrollo profesional & 0,499 & 3,30 & 0,01 \\
\hline 5 & Apoyo al emprendimiento & 0,583 & 3,21 & $-0,06$ \\
\hline 6 & Equipos y distribución de personas y material & 0,822 & 2,97 & $-0,26$ \\
\hline 7 & Organización y desempeño & 0,842 & 3,06 & $-0,19$ \\
\hline 8 & Estabilidad laboral & 0,826 & 3,12 & $-0,14$ \\
\hline 9 & Comunicación y desarrollo & 0,892 & 3,26 & $-0,02$ \\
\hline \multirow[t]{5}{*}{10} & Autonomía y profesionalismo & 0,657 & 3,73 & 0,36 \\
\hline & Alfa de Cronbach del Instrumento con 44 Elementos & 0,950 & & \\
\hline & Media general & & 3,29 & \\
\hline & Varianza general & & 1,49 & \\
\hline & Desviación estándar general & & 1,22 & \\
\hline
\end{tabular}




\section{Dimensiones de mayor y menor impacto en el clima organizacional}

Los valores promedio de las dimensiones de clima organizacional que se detallan en la Tabla 1, muestran bastantes similitudes ubicándose alrededor de los 3 puntos Likert. El promedio alcanza a 3,29 puntos con una desviación típica de 1,22.

La dimensión Autonomía y Profesionalismo (3,73 / o,36 estándar) presenta la mayor valoración, puesto que se valoran los estudios académicos y se reconoce la importancia y el profesionalismo de los trabajadores de APS en su quehacer habitual.

Las dimensiones por bajo la media fueron Equipos y Distribución de Personas y Material (2,97 / -o,26 estándar), lo cual concuerda con estudios realizados en Chile sobre Clima organizacional en Hospitales de Alta complejidad (Bustamante, et al., 2015) y que deriva del déficit de materiales e insumos que impide realizar una adecuada atención a los usuarios y pacientes, en términos de calidad, productividad y efectividad de los servicios de salud prestados a los usuarios (Santa Eulalia et al., 2012).

Por otra parte, las dimensiones Motivación Laboral (3,57 / o,23 estándar), Colaboración y Buen Trato (3,54/ o,20 estándar) y Desarrollo profesional (3,30 / o,o1 estándar), con valores por encima del promedio expre- san la relevancia que los trabajadores de la salud le otorgan a la perseverancia en el trabajo diario y el trabajo en equipo como ejes fundamentales de conformidad y aceptación.

En el lado negativo están las dimensiones Organización y Desempeño $(3,06 /-0,19)$, Estabilidad Laboral $(3,12 /-0,14)$, Iniciativa participativa $(3,26 /-0,02)$, Apoyo al emprendimiento $(3,21 /-0,06)$ y Comunicación y desarrollo (3,26/-0,02), evidenciando la disconformidad que genera el bajo número de personas contratadas por la institución y la falta de reconocimiento que perciben, además de las carencias que perciben en términos de apoyo a la iniciativa participativa, intra-emprendimiento y en los sistemas internos de comunicación por parte de sus superiores.

\section{Validez convergente y discriminante}

La validez convergente (Meliá, et al., 1990) se determinó mediante el Análisis de Factores Exploratorios AFE lo que permitió obtener los agrupamientos de ítems para la conformación de dimensiones, con previa verificación de pertinencia de la Medida de adecuación muestral de Kaiser-Meyer-Olkin (KMO) y Prueba de Esfericidad de Bartlett, lográndose un Índice KMO cercano a o,96 y esfericidad (p) significativa. La tabla 2 muestra la conformación de dimensiones de clima organizacional en APS.

Tabla 2 Análisis de Componentes principales.

\begin{tabular}{lcc}
\hline \multicolumn{1}{c}{ Factores } & \% de la varianza & \% acumulado \\
\hline 1. Organización y Distribución de Recursos & 43,367 & 43,367 \\
2. Colaboración y Buen Trato & 6,553 & 49,920 \\
3. Apoyo Mutuo y Emprendimiento & 5,368 & 55,289 \\
4. Motivación Laboral & 4,420 & 59,708 \\
5. Ambiente Laboral & 2,840 & 62,548 \\
6. Estabilidad Laboral & 2,799 & 65,347 \\
7. Autonomía y Profesionalismo & 2,466 & 67,813 \\
\hline \multicolumn{3}{c}{} \\
\hline
\end{tabular}

Tal como se aprecia en la Tabla 2, se verificó la validez discriminante por medio de las varianzas explicadas de los factores y las cargas factoriales de los componentes respecto de cada factor concentrando los ítems en 7 dimensiones que acumulan una varianza de $67,8 \%$, incluyendo 30 reactivos validados en APS y que, por su naturaleza, impactan en los respectivos ambientes de trabajo como se describe en la Tabla 3.

\section{Interpretación de factores}

La tabla 3 presenta los 7 factores determinados con el descriptor de sus respectivos reactivos. Se observa además que, en general, los ítems muestran cargas factoriales altas $(\geq 0,6)$ lo cual significa que efectivamente las afirmaciones que conforman los reactivos, contribuyen a darle sentido y contenido a los respectivos factores a los cuales pertenecen y colaboran a explicar la varianza parcial de cada uno de ellos y la total del constructo de clima organizacional (CCOV) utilizado en esta investigación.

El Factor 1 Organización y Distribución de Recursos, refiere a la productividad de los factores productivos, misma que se manifiesta en coherencia, eficacia y eficiencia de las prestaciones de salud, confirmando que si son estructurados de manera pertinente, son claves para el logro de resultados. Muestra que la organización y planificación de las obligaciones, así como la división de tareas, 
funciones y responsabilidades, deben coexistir con un sistema de recompensas y estímulos adecuados para fomentar el buen desempeño.

El Factor 2 Colaboración y Buen Trato, establece como punto relevante, que la colaboración entre pares, ayuda, apoyo y trato agradable de los directivos genera un clima organizacional que influye positivamente en la motivación y en el comportamiento de los funcionarios, promueva las relaciones humanas y consolida las confianzas mutuas en la organización.

Factor 3 Apoyo Mutuo y Emprendimiento, promueve y estimula la generación de nuevas ideas como componente clave del desempeño que los funcionarios aprecian al contar con apoyo directivo y al percatarse de que se les trata con respeto y confianza.

El Factor 4 Motivación Laboral, subraya el compromiso de los trabajadores por realizar un trabajo eficiente $y$, por lo mismo, transforma esta variable en un pilar fundamental del clima organizacional.
El Factor 5, Ambiente Laboral, manifiesta la importancia que los trabajadores de la salud asignan a los sistemas formales de comunicación entre personas, servicios y unidades para que trabajen de forma articulada y armónica.

Por su parte, el Factor 6 Estabilidad Laboral, representa la valoración que los funcionarios hacen de la seguridad del empleo, su pertenencia a una unidad de trabajo, y que los empleados perciben cómo esto influye en el compromiso individual y colectivo de para con la organización.

Por último, el Factor 7 Autonomía y Profesionalismo, pone de manifiesto el valor de la libertad, la valoración de la autonomía y la necesidad de los funcionarios de contar con espacio y atribuciones, por una parte, que les permitan ejercer con independencia sus talentos y, por otra, que se reconozca el valor de la experticia, el dominio contextual y calidad de sus prestaciones de salud.

Tabla 3 Reactivos de los factores determinados

\begin{tabular}{|c|c|c|}
\hline \multicolumn{2}{|r|}{ Organización y Distribución de Recursos } & \multirow{2}{*}{$\frac{\text { Cargas factoriales }}{0,709}$} \\
\hline 1 & En esta institución a veces se sabe bien quien tiene que decidir las cosas. & \\
\hline 2 & En esta institución hay disponibilidad de materiales del tipo y cantidad requeridos. & 0,701 \\
\hline 3 & Existe una buena comunicación entre la dirección y los trabajadores. & 0,690 \\
\hline 4 & La capacidad del funcionario es el criterio básico para asignar tareas en esta institución. & 0,673 \\
\hline 5 & $\begin{array}{l}\text { La distribución del personal y de material en esta institución permite que se realice un trabajo } \\
\text { eficiente y efectivo }\end{array}$ & 0,670 \\
\hline 6 & Todo lo que hay que hacer está claro, porque se nos explica bien y oportunamente. & 0,670 \\
\hline 7 & En esta institución los trabajos están bien asignados y organizados. & 0,644 \\
\hline 8 & $\begin{array}{l}\text { En esta institución los que se desempeñan mejor en su trabajo obtienen un mayor } \\
\text { reconocimiento de parte de sus superiores. }\end{array}$ & 0,642 \\
\hline 9 & $\begin{array}{l}\text { El número de personas que trabaja en esta institución es apropiado para la cantidad de trabajo } \\
\text { que se realiza. }\end{array}$ & 0,620 \\
\hline \multicolumn{2}{|r|}{ Colaboración y Buen Trato } & \\
\hline 10 & Los jefes entregan información a su personal respecto de su desempeño laboral. & 0,741 \\
\hline 11 & Cuando tengo que hacer un trabajo difícil puedo contar con la ayuda de mi(s) jefe(s). & 0,735 \\
\hline 12 & Los funcionarios saben lo que los supervisores esperan de ellos. & 0,677 \\
\hline 13 & Los jefes promueven las buenas relaciones humanas entre las personas de la institución. & 0,638 \\
\hline 14 & Los jefes de esta institución tratan con respeto a sus subordinados. & 0,625 \\
\hline & Los jefes son comprensivos cuando uno comete un error. & 0,617 \\
\hline \multicolumn{2}{|r|}{ Factor $3 \quad$ Apoyo Mutuo y Emprendimiento } & \\
\hline \multirow{2}{*}{$\begin{array}{l}16 \\
17 \\
\end{array}$} & El ambiente en esta institución es propicio para desarrollar nuevas ideas. & 0,625 \\
\hline & En esta institución la gente confía en los demás. & 0,622 \\
\hline \multicolumn{2}{|c|}{ Factor 4} & \\
\hline 18 & Las personas de esta institución se esfuerzan bastante por desarrollar eficientemente su labor. & 0,870 \\
\hline 19 & En esta institución la gente está comprometida con su trabajo. & 0,856 \\
\hline 20 & Las personas en esta institución muestran interés por el trabajo que realizan. & 0,843 \\
\hline \multicolumn{2}{|c|}{ Factor 5} & \\
\hline 21 & Esta institución se caracteriza por un ambiente de trabajo tenso. & 0,781 \\
\hline 22 & $\begin{array}{l}\text { Los trabajadores de esta institución creen más en el rumor que surge sobre un cierto hecho, } \\
\text { que en la información oficial. }\end{array}$ & 0,758 \\
\hline 23 & A veces trabajamos en forma desorganizada y sin planificación. & 0,737 \\
\hline 24 & En esta institución existen demasiadas críticas. & 0,735 \\
\hline 25 & En esta institución se nos mantiene desinformados sobre materias que deberíamos saber. & 0,673 \\
\hline \multicolumn{2}{|r|}{$\begin{array}{ll}\text { Factor } 6 & \text { Estabilidad Laboral } \\
\end{array}$} & \\
\hline 26 & $\begin{array}{l}\text { Me preocupan los efectos que puedan tener sobre la estabilidad de mi empleo los cambios de } \\
\text { método de trabajo y la automatización en esta institución. }\end{array}$ & 0,871 \\
\hline 27 & $\begin{array}{l}\text { Me preocupa que por una reorganización en la institución se vea afectada la estabilidad de mi } \\
\text { trabajo. }\end{array}$ & 0,858 \\
\hline 28 & Creo que en cualquier momento puedo perder el trabajo en esta institución. & 0,841 \\
\hline \multicolumn{2}{|r|}{ Autonomía y Profesionalismo } & \\
\hline & $\begin{array}{l}\text { Para que un trabajo de buenos resultados es necesario que sea hecho con un alto nivel de } \\
\text { conocimientos. }\end{array}$ & 0,631 \\
\hline & $\begin{array}{l}\text { En esta institución los jefes dan las indicaciones generales de lo que se debe hacer y se le deja } \\
\text { a los empleados la responsabilidad sobre el trabajo especifico }\end{array}$ & 0,626 \\
\hline \multicolumn{2}{|c|}{ Varianza total acumulada } & 67,813 \\
\hline
\end{tabular}




\section{Discusión}

Del análisis realizado y de la interpretación de los resultados obtenidos en este trabajo se puede afirmar que, en general, los 10 factores y los 44 reactivos del clima organizacional identificados originalmente para hospitales de alta complejidad, se concentran ahora en 7 factores y 30 ítems relevantes a nivel de atención primaria de salud, con una varianza explicada acumulada que alcanza el $67,8 \%$.

En primer lugar es preciso destacar lo indicado por Chiang, Martín y Núñez (2010) quienes a partir de un meta-análisis, afirman que el clima organizacional como instrumento general de gestión está presente en el 100\% de los estudios de gestión lo cual demuestra la significativa influencia de esta herramienta de dirección para la supervisión de las organizaciones.

Así mismo, es posible afirmar que algunas de las dimensiones del clima organizacional muestran alta relevancia. Entre estas se puede mencionar las dimensiones de Responsabilidad (también llamada Autonomía), Recompensa y Presión, Relaciones entre compañeros y Característica de la función (también llamada Estructura), consideradas todas entre las más relevantes relacionadas con la satisfacción laboral de los trabajadores según afirma Chiang, et al., (2010).

Por otra parte, sin perjuicio de que existen diversos estudios que relacionan el clima organizacional con la satisfacción laboral, continúa la controversia sobre si el clima afecta la satisfacción o viceversa (Payne, Pheysey \& Pugh, 1971; Puch, Ruiz \& Almeida, 2012), parece claro que ambas dimensiones se influyen mutuamente de manera recursiva y permanente.

Al respecto, las percepciones individuales de clima presentan relaciones significativas con distintas variables de carácter actitudinal, emocional o afectivo, como son la satisfacción laboral y diferentes dimensiones de ella, tales como el conflicto, la ambigüedad y la sobrecarga del rol, el bienestar afectivo, el compromiso con la organización y la propensión al abandono, como lo señalan diversos estudios en esta materia (Mañas, González \& Peiró, 1999; Patterson, et al., 2005; Peiró, Zurriaga \& González-Romá, 2002; Subirats, González-Romá, Peiró \& Mañas, 1997), por cuanto todas estas dimensiones son claves y pertinentes en las mediciones de clima organizacional, lo cual se confirma una vez más a través de los hallazgos realizados en el presente trabajo; como es el caso de los componentes que conforman las dimensiones Autonomía y Profesionalismo, Ambiente Laboral y Motivación Laboral.

Adicionalmente se confirma que la satisfacción laboral es una respuesta emocional positiva hacia el trabajo (Segura, 2012), mientras que la insatisfacción laboral es una respuesta emocional negativa que frustra o niega los valores de trabajo de la persona (Lisbona, Palací \& Gómez, 2008; Peiró \& Salvador, 1993; Senic \& Marinkovic, 2013). Ampliando el análisis del clima organizacional, se establece así mismo su influencia sobre diversos fenómenos laborales como es el establecimiento de metas y objetivos, el diseño de puestos de trabajo, los sistemas de recompensas, las características organizacionales, el liderazgo, la participación en la toma de decisiones, el perfil demográfico y los factores relacionados con las disposiciones y orientaciones conductuales pertinentes en una organización (Griffin \& Bateman, 1986), además de validar el uso de la tecnología empleada por una organización y que puede hacer variar, en forma positiva o negativa, el clima organizacional.

Se ratifica el hecho que Autonomía y profesionalismo y Colaboración y buen trato influyen el clima organizacional y que las mismas, en vinculación con el liderazgo, establecen un tipo de relaciones entre superiores y subordinados que puede constituirse en un factor relevante del clima (Pérez, Maldonado \& Bustamante, 2006; Poghosyan, etal., 2013; Puch et al., 2012).

Por otra parte se ratifica que el signo y dirección de las percepciones del clima dependerá de la congruencia que se produzca entre la estructura organizacional, los estilos de supervisión y los procesos de comunicación (Ezequiel, 2000; Mendizábal, 2003). Asimismo, se demuestra que los líderes influyen positivamente sobre las percepciones individuales de apoyo y de orientación a las reglas, contribuyen a la Autonomía y Profesionalismo de los personales y, en consecuencia, mejoran el Ambiente Laboral, de manera tal que parece evidente que estas dos dimensiones deben ser potenciadas por lo líderes, de forma que el clima se vea afectado positivamente e influya en la mejor disposición de los trabajadores (Peiró et al., 1993; Peiró, et al., 2002).

En relación con las dimensiones Iniciativa participativa y Apoyo al emprendimiento, se concluye que estas dimensiones efectivamente influyen sobre la Motivación Laboral y en consecuencia sobre el clima organizacional, confirmando que la innovación estimula la satisfacción y el mejor acoplamiento de las personas a su trabajo, especialmente cuando se les permite ser creativos y tomar decisiones propias en sus áreas de desempeño (D'Amato \& Burke, 2009; King, Hebl, George \& Matusik, 2010).

\section{Conclusiones}

Concordante con el análisis realizado, se concluye que a nivel de hospitales de alta complejidad y a nivel de atención primaria en APS, existe una percepción de desaprobación que afecta la dimensión Equipos y Distribución de Personas y Material, evidenciando el déficit de 
recurso humano que, como se constata en la práctica clínica, es una falencia clave de alto impacto en las prestaciones de una red de salud.

Se concluye además que en atención primaria y en hospitales de alta complejidad, las dimensiones que afectan el clima organizacional son dispares en cuanto a Estabilidad laboral, lo que se puede explicar por las diferencias que se observan en los escenarios en que operan ambos niveles de atención, el hospitalario más centralizado a nivel del $\mathrm{Mi}$ nisterio de Salud que establece sus directrices y la Atención Primaria de Salud APS, que más bien opera de manera descentralizada, territorialmente descentralizadas, bajo la administración de las autoridades municipales.

Porúltimo, seratifica elroldelos líderes quienes inciden positivamente sobre las conductas de apoyo y de orientación a las reglas y sobre la autonomía y profesionalismo, haciendo que estas dos dimensiones se potencien mutuamente, de forma tal que el clima mejore influyendo en la mejor disposición de los trabajadores

\section{Conflicto de intereses}

Los autores declaran no tener ningún conflicto de intereses.

\section{Referencias}

Acevedo, G., Farias, A., Sánchez, J., Astegiano, C., \& A., Fernendez (2012). Condiciones de trabajo del equipo de salud en centros de atención primaria desde la perspectiva del trabajo decente. ResearchGate, 3(12), 15-22.

Acevedo, G., Farias, A., Sánchez, J., Astegiano, C. \& Fernández, A. (2012). Condiciones de trabajo del equipo de salud en centros de atención primaria desde la perspectiva de trabajo decente. Revista Argentina de Salud Pública, 3(12), 15-22.

Bass del Campo, C. (2012). Family health model in Chile and greater resoluteness of primary health care: contradictory or complementary? Medwave, 12(11), e5571e5571. https://doi.org/10.5867/medwave.2012.11.5571

Bernal, I., Pedraza, N. A., \& Sánchez, I. (2015). El clima organizacional y su relación con la calidad de los servicios públicos de salud: diseño de un modelo teórico. Retrieved October 30, 2016, from http:// www.redalyc.org/articulo.oa? id $=21233043002$ Bustamante U. Miguel A., Opazo B. \& Pablo A. (2004). Hacia Un Concepto De Complejidad: Sistema, Organización Y Empresa. Serie Documentos Docentes FACE SDD, año 2, $\mathrm{N}^{\circ} 3.23 \mathrm{P}$.

Bustamante, M. A., Grandón, M. L. , \& Lapo, M. del C. (2015). Caracterización del clima organizacional en hospitales de alta complejidad en Chile. Estudios Gerenciales, 31(137), 432-440. https://doi.org/10.1016/j.estger.2015.08.003

Bustamante, M. A. Lapo, $M$. del C. \& Grandón, $M$. L. (2016). Creación de un cuestionario de clima organizacional para hospitales de alta complejidad, Chile. Gerencia Y Políticas de Salud, 15(30). https://doi.org/10.11144/Javeriana.rgyps15-30.ccco

Cardona, D. R., \& Zambrano, R. (2014). Revisión de instrumentos de evaluación de clima organizacional. Estudios Gerenciales, 30(131), 184-189. https://doi.org/10.1016/j.estger.2014.04.007

Carlfjord, S., Andersson, A., Nilsen, P., Bendtsen, P., \& Lindberg, M. (2010). The importance of organizational climate and implementation strategy at the introduction of a new working tool in primary health care. Journal of Evaluation in Clinical Practice, 16(6), 13261332. https://doi.org/10.1111/j.1365-2753.2009.01336.x

Carmona, V., \& Jaramillo, E. (2010). Estudio del cli- ma organizacional en la ESE Hospital San Jorge de Pereira: Cooperativa de Trabajo Asociado Multiser CTA, Profesalud CTA, Induaseo, Nutrisalud Risaralda Ltda, Sociedad Neuroquirurgica de Risaralda, Imágenes Diagnosticas (Thesis). Universidad Tecnológica de Pereira. Retrieved from http:// repositorio.utp.edu.co/dspace/handle/11059/2230

CEPAL (2012). Las personas mayores en América Latina y el Caribe Departamento de Asuntos Económicos y Sociales (DESA), Naciones Unidas; CEPAL. 2012; https://goo.gl/ XRZgPx ; https://goo.gl/e4Uzyj (Accessed on 12/12/2017)

chiang, M., Martín, M. J. \& Núñez, A. (2010). Relaciones entre el clima organizacional y la satisfacción laboral. Universidad Pontifica Comillas. Madrid

Chiang, M., Salazar, C., Martín, P. \& Núñez, A. (2011). Clima organizacional y satisfacción laboral: Una comparación entre hospitales públicos de alta y baja complejidad. Salud de Los Trabajadores, 19(1), 05-16.

Cújar, A., Ramos, C., Hernández, H. \& López, J. (2014). Cultura organizacional: evolución en la medición. Estudios Gerenciales, 29(128), 350-355.

D'Amato, A. \&, Burke M. J. (2009). Psychological and organizational climate research: Contrasting perspectives and research traditions. A special issue of the European Journal of Work and Organizational Psychology. Londres: Psychology Press.

Eyssautier M. 2006. Metodología de la investigación. México D.F.: Editorial Thomson; 2006.

Ezequiel A. (2000). El valor de las redes, los canales y las comunicaciones informales en la empresa. Editorial Gestión 2000. 2002; 06:33-45.

González-Burboa, A., Manríquez, C. \& Venegas, M. (2014). Clima Organizacional en una Dirección de Administración de Salud Municipal. Ciencia \&amp; Trabajo, 16(51), 152-157. https://doi.org/10.4067/S0718-24492014000300005

Greenslade, J. H. \& Jimmieson, N. L. (2011). Organizational factors impacting on patient satisfaction: A cross sectional examination of service climate and linkages to nurses' effort and performance. International Journal of Nursing Studies, 48(10), 1188-1198. https://goo.gl/wEqqoB

Griffin,R.W.-Bateman T.S. (1986): Job satisfacation and or ganizacional commitment. En C.L. Cooper y I. Robertson (eds.). International review Industrial and Organizational Psychology. New York: John Wiley and sons.

Hernández R., Fernández C. \& Baptista P. (2006). Metodología de la Investigación (4.a ed.) McGraw-Hill, México D. F. Retrieved from https://goo.gl/mRZLii

INE. 2014. Población, país y regiones: actualización población 2002-2012 y proyección 2013-2020. 2014. Disponible en: https://goo.gl/DhVfWf (Accessed on 12/12/2017)

Jiménez, R. (2004). Indicadores de calidad y eficiencia de los servicios hospitalarios. Una mirada actual. Retrieved October 30, 2016, from http://www. bvs.sld.cu/revistas/spu/vol30 1 04/spo4104.htm

King, E. B., Hebl, M. R., George, J. M. \&, Matusik, S. F. (2010). Understanding tokenism: Antecedents and consequences of a psychological climate of gender inequity. Journal of Management 36 (2): 482-510.

Ley 17.374 (1970). Ley-17374 10-Dic-1970 Ministerio De Economía - Ley Chile - Biblioteca del Congreso Nacional. Retrieved October 31, 2016, from https://goo.gl/6UcTbF

Ley-19.628 (1999). LEY-19628 28-AGO-1999 Ministerio Secretaría General De La Presidencia-Ley Chile-Biblioteca del Congreso Nacional. Retrieved November 2, 2016, from https://www.leychile.cl/Navegar?idNorma $=141599$

Lisbona A. , F. Palací \&, Gómez, A. (2008). Escala de clima para la iniciativa y para la seguridad psicoló gica: adaptación al castellano y su relación con el desempeño organizacional. Revista de Psicología del Trabajo y de las organizaciones, 24 (2), 153-167

Mañas A. , V. González \& J. Peiró (1999). El clima de los equipos de trabajo: determinantes y consecuencias. (1.a ed.), Universidad de Almería, Servicio de Publicaciones, D.L, Almería (1999)

Meliá, J.L., Pradilla, J.F., Martí, N., Sancerni, M.D., Oliver, A. \& Tomás J.M. (1990). Estructura factorial, fiabilidad y validez del Cuestionario de Satisfacción S21/26: Un instrumento con formato dicotómico orientado al trabajo profesional. Revista de PsicologiaUniversitas 
Tarraconensis, 12(1/2), 25-39. Retrieved from http:// www.uv.es/ meliajl/Research/Art Satisf/ArtS21_26. PDF

Mendizábal L. (2003). Reglas de oro para una comunicación efectiva. Mecanismos de influencia para obtener resultados. Editorial Trillas 2003; 06:7-25.

MINSAL (2016). Informe sobre brechas de personal de salud por servicio de salud. (n.d.) Retrieved from https://goo.gl/8SXdNs

Neal, A., Griffin, M. A. \& Hart, P. M. (2000). The im pact of organizational climate on safety climate and individual behavior. Safety Science, 34(1-3), 99-109. https://doi.org/10.1016/Sog25-7535(oo)oooo8-4

Noboa, A. (2007). Especificidades del clima organizacional en las instituciones de salud. Macau: Universidad de la República de Uruguay. https://goo.gl/E73dXZ

OMS (2006). Informe sobre la salud en el mundo 2006. Retrieved from http://www.who.int/whr/2006/whro6_es.pdf

OMS (2008). Declaración de Kampala y prioridades para la acción internacional. Organización Mundial de la Salud. Retrieved from http://www.who.int/iris/handle/10665/7783

Oviedo, H. C. \& Campo-Arias, A. (2005). An Approach to the Use of Cronbach's Alfa. Revis ta Colombiana de Psiquiatría, 34(4), 572-580.

Patarroyo, J. C. (2012). Clima organizacional: elemento clave para el proceso de calidad en las instituciones prestadoras de salud (other). Universidad Nacional de Colombia. Retrieved from http://www.bdigital.unal.edu.co/759o/

Patterson M, West M, Shackleton V, Dawson J, Lawthom R, Maitlis S, D. L. Robinson \& Alison M. Wallace (2005). Validating the organizational climate measure: Links to managerial practices, productivity and innova tion. Journal of Organizational Behavior. 26: 379-408.

Payne RL, Pheysey DC, Pugh DS. (1971). Organization struc ture, organizational climate and group structure: an explanatory study of their relationship in two British ma nufacturing companies. Occup Psychol. 1971; 45: 45-56

Peiró, J.M. \& Salvador, A. (1993) Control del Estrés Laboral. Madrid, Eudema.

Peiró, J.M., Zurriaga, R. \& González-Romá, V. (2002). Análisis y diagnóstico de las situaciones y experiencias de estrés colectivo en las unidades de trabajo y en las organizaciones de servicios sociales. Revista del INSHT, 20, 11-20.

Pelaes, O. (2010). Relación entre el clima organizacional y la satisfacción del cliente en una empresa de servicios telefónicos. Universidad Nacional Mayor de San Marcos. Programa Cybertesis PERÚ. Retrieved from http:// cybertesis.unmsm.edu.pe/handle/cybertesis/1140

Pérez, I., Guillén-Grima, F., Brugos, A., Aguinaga, I. \& Fernández-Martínez, C. (2012). Validación de un cuestionario de mejora de la satisfacción labora (CMSL) en profesionales de atención primaria. Anales Del Sistema Sanitario de Navarra, 35(3), 413-423.

Pérez, I., Maldonado, M. \& Bustamante, S. (2006). Clima organizacional y gerencia: inductores del cambio organizacional. Investigación Y Postgrado, 21(2), 231-248

Pettigrew, A. M. (1979). On Studying Organizational Cultures. Administrative Science Quarterly, 24(4), 570-581. https://doi.org/10.2307/2392363

Poghosyan, L., Nannini, A. \& Clarke, S. (2013). Organizational climate in primary care settings: implications for nurse practitioner practice. Journal of the American Association of Nurse Practitioners, 25(3),
134-140. https://doi.org/10.1111/j.1745-7599.2012.00765. Poghosyan, L., Nannini, A., Finkelstein, S. R., Mason, E \& Shaffer, J. A. (2013). Development and psychometric testing of the Nurse Practitioner Primary Care Organizational Climate Questionnaire. Nursing Research, 62(5), 325-334. https://doi.org/10.1097/NNR.obo13e3182a131d2 Puch M, Ruiz D, \& Almeida C. (2012). Diagnóstico del clima organizacional del Hospital de la Mujer Zacatecana. Revista Salud Pública y Nutrición. 2012; 13 (4): 1-8. http://www.respyn.uanl. $\mathrm{mx} / \mathrm{xiii} / 4 /$ articulos/clima_organizacional.htm Rodríguez, A., Álvarez, A., Sosa, I., De Vos, P., Gorbea, B., H, M. \& Van der Stuyft, P. (2010). Inventario del clima organizacional como una herramienta necesaria para evaluar la calidad del trabajo. Revista Cubana de Higiene Y Epidemiología, 48(2), 177-196.

Santa Eulalia, J. \& Sampedro, B. (2012). Clima organizacional en instituciones de atención primaria de salud. Revista Médica Electrónica, 34(5), 606-619.

Segredo Pérez, Alina María, García Milian, Ana Julia, López Puig, Pedro, León Cabrera, Pablo \& Perdomo Victoria, Irene. (2015). Systemic organizational climate approach and its implementation in the public health care. Revista Cubana de Salud Pública, 41(1) Recuperado en 20 de diciembre de 2016, de https://goo.gl/MZKJZK

Segredo, A. (2013). Clima organizacional en la gestión del cambio para el desarrollo de la organización. Revista Cubana de Salud Pública, 39(2), 385-393.

Segredo, A. \& Reyes, D. (2004). Clima organizacional en salud pública. Consideraciones generales. Retrieved October 30, 2016, from http://www.cocmed.sld.cu/no83/n83rev4.htm

Segura A. (2012). Clima organizacional: un modo eficaz para dirigir los servicios de salud. Avances en Enfermería. 2012; 30 (1): 107-13.

SENAMA (2013). Tercera Encuesta Nacional Calidad de Vida en la Vejez. Chile y sus Mayores. 2013; https://goo.gl/Svizj9 (Accessed on 12/12/2017)

Senic V. \& V. Marinkovic (2013). Patient care, satisfaction and service quality in health care International Journal of Consumer Studies, 37 (3) (2013), pp. 312-319

Subirats, M., González-Romá, V., Peiró, J.M. \& Mañas, M.A.1997). Concequences of worteam climate. 11 FOCUS Symposium, 20-22 Sep., Croacia.

Téllez, Á. (2016). Atención Primaria: Factor clave en la reforma al sistema de salud. Centro UC Políticas Públicas. Retrieved October 30, 2016, from https://goo.gl/GC5QdG Urquiza R. (2012). Satisfacción y calidad del servicio de salud. Revista Médica La Paz, 18 (2) (2012), pp. 65-69

Vega, D., Arévalo, A., Sandoval, J., Aguilar, M. C. \& Giraldo, J. (2006). Panorama sobre los estudios de clima organizacional en Bogotá, Colombia(1994-2005). Retrieved October 30, 2016, from http://www.redalyc.org/articulo.oa?id=67920212

Vignolo, J., Vacarezza, M., Âlvarez, C. \& Sosa, A. (2011). Niveles de atención, de prevención y atención primaria de la salud. Archivos de Medicina Interna, 33(1), 7-11.

Walpole R., Myers R., \& Myers S. (1999). Probabilidad y estadística para ingenieros (6.a ed.) Prentice-Hall, Hispanoamericana S.A, México. Retrieved from https://goo.gl/UKVMnx 